\title{
Characteristics of NDM-1-producing Klebsiella pneumoniae ST234 and ST1412 isolates spread in a neonatal unit
}

\author{
Xu Huang ${ }^{1,2+}$, Xiangjun Cheng ${ }^{1,2+}$, Pengfei Sun ${ }^{1,2}$, Chenjie Tang ${ }^{1,2}$, Fang $\mathrm{Ni}^{1,2}$ and Genyan Liu ${ }^{1,2^{*}}$ (D)
}

\begin{abstract}
Background: The emergence of carbapenem-resistant Klebsiella pneumoniae (CR-KP) has become a significant problem worldwide and also being a major threat to children and newborns. Here we report an outbreak of NDM-1-producing $K$. pneumoniae in a neonatal unit.

Results: Six CR-KP strains, isolated from neonates with symptoms of infection, were identified using a VITEK-2 compact system, and the clinical data were retrieved from the electronic case records. In vitro susceptibility testing with broth dilution method showed that all six K. pneumoniae isolates were resistant to carbapenems and susceptible to colistin, aminoglycosides, fluoroquinolones and tigecycline. Based on the polymerase chain reaction results, each isolate was found to be bla $a_{\mathrm{NDM}-1}$ gene positive. Clonal relationships were analysed using pulsed-field gel electrophoresis (PFGE) and multilocus sequence typing (MLST) and showed that two different PFGE patterns were formed, which belonged to sequence types ST234 and ST1412. Plasmids carrying bla NDM-1 $_{1}$ were successfully transferred from four of the six isolates to an Escherichia coli recipient through conjugative assays. S1-PFGE and Southern blot hybridization showed that four NDM-1-producing K. pneumoniae were clonal and carried bla $a_{\mathrm{NDM}-1}$ on the same plasmid. The outbreak was effectively controlled by reducing the potential infection sources. All the patients were successfully treated and recovered after receiving an increased dose of carbapenems. Although the source of this outbreak was not clear, comprehensive measures were carried out and the outbreak was effectively controlled.
\end{abstract}

Conclusions: ST234 and ST1412 of NDM-1-producing Klebsiella pneumoniae are the resistant clone spread in the neonatal unit, comprehensive infection control measures and optimized carbapenem therapy played an important role in controlling this NDM-1-producing K. pneumoniae outbreak.

Keywords: Multidrug-Resistant, carbapenem-resistant Klebsiella pneumoniae, bla $a_{\mathrm{NDM}-1,1}$ Neonate, Outbreak

\section{Background}

Carbapenem-resistant Enterobacteriaceae (CRE) has become more and more serious in hospital-acquired multi-drug resistant infections [1]. Patients with CRE infection usually face limited treatment options, prolonged hospitalization, increased economic burden, and even increased mortality. Carbapenem-resistant Klebsiella pneumoniae (CR-KP) is one of the most popular CRE in hospitals around the world [2]. Carbapenemases are the main mechanism of carbapenem resistance in CRE,

\footnotetext{
* Correspondence: liugenyan@jsph.org.cn

${ }^{\dagger}$ Xu Huang and Xiangjun Cheng contributed equally to this work.

'Department of Laboratory Medicine, the First Affiliated Hospital with Nanjing Medical University, Nanjing 210029, People's Republic of China

${ }^{2}$ National Key Clinical Department of Laboratory Medicine, Nanjing 210029,

People's Republic of China
}

including KPC, NDM, IMP, VIM, OXA-48, NMC, GES and SME enzymes [3]. Among them, NDM-1 gene is a novel metallo-beta-lactamase that was first reported in a Swedish patient traveled to New Delhi in 2008 [4]. Since then, Sweden, United Kingdom, Belgium, China, Japan, France, Austria, Germany, Norway, Netherland, Australia and Canada have also reported NDM-1-producing isolates [5].

Since the first K. pneumonia isolates harboring $b l a_{N D M-1}$ were reported in Nanchang, China in 2013, they have spread rapidly in China. Even outbreaks of NDM-1 producing $K$. pneunoniae were also reported in Shanghai, Yunnan, Hunan and so on [6-8]. For a long time, CRE infections have mainly come from adult patients. In recent years, the outbreak of CRE infection in newborns has been reported and has attracted more and more attention $[6,9]$. 
For different care model and limited drug selection for neonates, effective infection control and treatment should be further studied [10]. Here, we present an outbreak of NDM-1-producing $K$. pneumoniae in the neonatal ward of a university hospital in China. The molecular characteristics and transmission mechanism of NDM-1-producing $K$. pneumoniae was also studied.

\section{Methods}

\section{Bacterial isolates and patients}

K. pneumoniae strains were collected from neonates with symptoms of infection who were admitted to the neonatal ward of a university hospital in Nanjing between June and August of 2016. Identification and in vitro susceptibility tests were carried out and carbapenem-resistance was analyzed with VITEK-2 compact system (bioMérieux, Marcy-l'Étoile, France). The electronic case records including patient demographics, antimicrobial treatment and clinical outcomes were retrieved and reviewed. In order to investigate the source of nosocomial infection, related specimen were collected from incubator surface, head circumference tape, blood machine surface and healthcare worker, isolates named KP7 to KP10 were analyzed.

\section{Antimicrobial susceptibility testing}

The minimum inhibitory concentration (MICs) of imipenem (IPM), meropenem (MEM), ceftazidime (CAZ), cefepime(FEP), aztreonam (ATM), piperacillin /tazobactam (TZP), sulbactam/cefoperazone (SCF), amikacin $(\mathrm{AM})$, piperacillin (PIP), levofloxacin (LE), polymixin $B$ (PB) and tigecycline (TG) were determined using the broth micro dilution method. Two quality control (QC) strains, Escherichia coli ATCC 25922 and K. pneumoniae ATCC 700603 were used. The results of the antimicrobial susceptibility testing were interpreted as specified by the Clinical and Laboratory Standards Institute (CLSI) [11], except for sulbactam/cefoperazone, colistin and tigecycline, which were interpreted in accordance with the European Committee on Antimicrobial Susceptibility Testing clinical breakpoints (version 6.0) [12].

\section{Resistant gene detection}

PCR was performed as previously described to detect carbapenem resistance genes (bla $a_{\mathrm{KPC}}, b l a_{\mathrm{IMB}}$ bla $a_{\mathrm{VIM}}, b l a_{\mathrm{NDM}}$ and $\left.b l a_{\text {OXA-48 }}\right)$, common extended-spectrum $\beta$-lactamase (ESBL) genes (bla $a_{\mathrm{CTX}-\mathrm{M}-1 \mathrm{G}}, b l a_{\mathrm{CTX}-\mathrm{M}-2 \mathrm{G}}, b l a_{\mathrm{CTX}-\mathrm{M}-8 \mathrm{G}}$, $b l a_{\mathrm{CTX}-\mathrm{M}-9 \mathrm{G}}, b l a_{\mathrm{TEM}}, b l a_{\mathrm{OXA}-1}$ and $\left.b l a_{\mathrm{SHV}}\right)$, plasmid mediated quinolone resistance (PMQR) genes (qnrA, qnrB, qnrS, and aac-(6')-Ib-cr) and $16 \mathrm{~S}$ rRNA methylase genes $(\operatorname{arm} A, r m t B)$ in all strains [13-15]. PCR amplicons were sequenced, and the sequencing results were compared to the database at the National Center for Biotechnology
Information (NCBI) (https://www.ncbi.nlm.nih.gov/gen bank/) using BLAST searches.

\section{Molecular typing}

NDM-1-producing strains were genotyped using PFGE and MLST [16]. The allelic profiles and sequence types (ST) were available in the MLST database (http:// bigsdb.pasteur.fr/klebsiella/klebsiella.html). Clonal relationships were analysed using PFGE and bacterial DNA was digested with XbaI endonuclease (TaKaRa, Dalian, China) as previously described [7]. Salmonella enterica serotype H9812 was used as a marker. The PFGE patterns were compared using BioNumerics software (Applied Maths, Kortrijk, Belgium), and a phylogenetic tree was built for cluster analysis. Clusters were defined as DNA patterns sharing $>90 \%$ similarity.

\section{Conjugation experiments}

Conjugative assays were performed using sodium azide-resistant $E$. coli J53 as the recipient strain (donated by professor Wenen Liu, Central South University) [17]. Transconjugants were selected on MacConkey agar plates containing $150 \mathrm{mg} / \mathrm{L}$ sodium azide (Sigma Aldrich, St Louis, MO) and $30 \mathrm{mg} / \mathrm{L}$ ceftazidime (Sigma-Aldrich) for $24 \mathrm{~h}$ at $35^{\circ} \mathrm{C}$. Presence of the $b l a_{\mathrm{NDM}-1}$ gene in the transconjugants was confirmed by PCR analysis, and their antimicrobial susceptibilities were determined as well.

\section{Plasmid analysis}

S1-PFGE and Southern blotting were performed to analyse the size of the NDM-1-carrying plasmids in the $K$. pneumoniae strains as previously described and the results were analyzed according to the criteria proposed by Tenover et al. $[18,19]$ Plasmid DNA of the isolates embedded in agarose gel plugs were digested with S1 nuclease and separated by PFGE. Plasmids obtained by PFGE were then transferred to a positively charged nylon membrane. The membrane was hybridized with digoxigenin-labelled $b l a_{\mathrm{NDM}-1}$-specific probes and the signals were detected using an NBT/BCIP colour detection kit (Roche Applied Sciences, Penzberg, Germany).

\section{Results}

\section{Clinical characteristics of the $K$. pneumoniae isolates}

The clinical profiles of the six NDM-1-positive $K$. pneumoniae isolates (KP1 to KP6) are shown in Table 1, including the patient demographics, the date of specimen isolation, specimen source and clinical diagnosis, antimicrobial treatment and clinical outcomes. The six NDM-1-positive $K$. pneumoniae isolates were obtained from blood $(n=5)$ and sputum $(n=1)$. Five patients had sepsis, and one had neonatal respiratory distress syndrome. No infants died from their infections after effective treatment. The newborns experienced long 
Table 1 Clinical profiles of the six NDM-1-positive K. pneumoniae isolates

\begin{tabular}{|c|c|c|c|c|c|c|c|c|}
\hline Case & Sex & $\begin{array}{l}\text { Pregnancy } \\
\text { duration (wk) }\end{array}$ & Sample type & Type of infections & Antimicrobial therapy & PFGE type & ST type & $\begin{array}{l}\text { NDM-coding } \\
\text { plasmids (Kb) }\end{array}$ \\
\hline 1 & $\mathrm{~F}$ & 32 & Blood & $\begin{array}{l}\text { Neonatal sepsis, neonatal } \\
\text { respiratory distress syndrome }\end{array}$ & meropenem $^{a}$ & A & 234 & none \\
\hline 2 & $\mathrm{~F}$ & 31 & Blood & $\begin{array}{l}\text { Neonatal sepsis, neonatal } \\
\text { respiratory distress syndrome }\end{array}$ & meropenem & A & 234 & none \\
\hline 3 & M & 26 & Sputum & $\begin{array}{l}\text { Neonatal respiratory distress } \\
\text { syndrome }\end{array}$ & imipenem ${ }^{\mathrm{b}}$ & B & 1412 & 50 \\
\hline 4 & M & 30 & Blood & $\begin{array}{l}\text { Neonatal sepsis, neonatal } \\
\text { respiratory distress syndrome }\end{array}$ & meropenem & B & 1412 & 50 \\
\hline 5 & M & 35 & Blood & $\begin{array}{l}\text { Neonatal sepsis, neonatal } \\
\text { pneumonia }\end{array}$ & meropenem & B & 1412 & 50 \\
\hline 6 & M & 29 & Blood & Neonatal sepsis & meropenem & B & 1412 & 50 \\
\hline
\end{tabular}

${ }^{a}$ meropenem $0.5 \mathrm{~g} \mathrm{IVD,qd;}{ }^{\mathrm{b}}$ imipenem $1.0 \mathrm{IVD}$ qd;

hospitalization periods (mean, 62 days), and treatment with increased doses of carbapenem showed good prognoses.

\section{Antibiotic susceptibility testing and characteristics of drug-resistant genes}

All six clinical isolates were resistant to imipenem, meropenem, ceftazidime, cefepime, piperacillin, piperacillin /tazobactam, sulbactam/cefoperazone and aztreonam (except for KP6, MIC $=0.125 \mathrm{mg} /$ liter) but remained susceptible to amikacin, levofloxacin, polymixin B and tigecycline. The transconjugants had similar susceptibility results. Among the six NDM-1-positive isolates, $67 \%(4 / 6)$ co-harboured bla $a_{\mathrm{SHV}-148}$ and $33 \%(2 / 6)$ co-harboured $b l a_{\mathrm{CTX}-\mathrm{M}-14} b l a_{\mathrm{SHV}-27}$ and qnrB4. Other resistance genes were not detected. Results of antibiotic susceptibility testing, drug-resistant genes analysis of clinical isolates and respective transconjugants are shown in Table 2.

\section{Molecular epidemiology}

Two distinct PFGE patterns were obtained from the XbaI DNA digests among the six CR-KP isolates: type A $(n=2)$ and type B $(n=4)$. Carbapenem-susceptible K. pneumoniae isolated from the incubator surface, head circumference tape, blood machine surface, and medical workers showed no homology with the six CR-KP isolates (Fig. 1a). Two distinct sequence types were obtained from the six isolates:KP1 together with KP2 are ST234 and KP3, KP4, KP5 and KP6 are ST1412. Comparison of these results showed that all PFGE type A isolates corresponded to ST234, and the type B isolates corresponded to ST1412(Fig. 1b).

\section{Transfer of carbapenemase resistance}

Transconjugants were obtained in the conjugation experiments, with a success rate of $67 \%$. In the conjugation experiments, the $b l a_{\mathrm{NDM}-1}$ genes were transferred successfully from the donor $K$. pneumoniae isolates (except KP1 and KP2 isolates) to the recipient E. coli J53 via plasmids. Antimicrobial susceptibility detection showed

Table 2 Antimicrobial susceptibilities of the six NDM-1-positive K. pneumoniae isolates

\begin{tabular}{|c|c|c|c|c|c|c|c|c|c|c|c|c|c|}
\hline \multirow{2}{*}{$\begin{array}{l}\text { Isolate } \\
\text { No. }\end{array}$} & \multirow[t]{2}{*}{ Resistance mechanisms } & \multicolumn{12}{|c|}{$\mathrm{MIC}\left(\mathrm{mg} / \mathrm{liter}\right.$ of $^{\mathrm{a}}$} \\
\hline & & SCF & IPM & CAZ & MEM & TZP & PB & FEP & AM & PIP & LE & TG & ATM \\
\hline KP1 & NDM-1, CTX-M-14, SHV-27,qnrB4 & $>128$ & 64 & $>128$ & 64 & $>128$ & 0.5 & 64 & 0.125 & $>128$ & $<0.125$ & 0.125 & 4 \\
\hline KP2 & NDM-1, CTX-M-14, SHV-27,qnrB4 & $>128$ & 64 & $>128$ & 64 & $>128$ & 0.5 & 64 & 0.125 & $>128$ & $<0.125$ & $<0.125$ & 4 \\
\hline KP3 & NDM-1, SHV-148 & $>128$ & 32 & $>128$ & $>128$ & $>128$ & 2 & $>128$ & 0.25 & $>128$ & $<0.125$ & 0.25 & 4 \\
\hline KP4 & NDM-1, SHV-148 & $>128$ & 32 & $>128$ & $>128$ & $>128$ & 1 & $>128$ & 0.25 & $>128$ & $<0.125$ & $<0.125$ & 4 \\
\hline KP5 & NDM-1, SHV-148 & $>128$ & 32 & $>128$ & $>128$ & $>128$ & 1 & 64 & 1 & $>128$ & $<0.125$ & $<0.125$ & 8 \\
\hline KP6 & NDM-1, SHV-148 & $>128$ & 32 & $>128$ & 32 & $>128$ & 1 & 32 & 1 & $>128$ & $<0.125$ & $<0.125$ & 0.125 \\
\hline$J 53-3$ & NDM-1 & $>128$ & 16 & $>128$ & 32 & $>128$ & 2 & 128 & 8 & $>128$ & $<0.125$ & $<0.125$ & 0.25 \\
\hline J53-4 & NDM-1 & $>128$ & 32 & $>128$ & 32 & $>128$ & 2 & 64 & 4 & $>128$ & $<0.125$ & $<0.125$ & 0.25 \\
\hline$J 53-5$ & NDM-1 & $>128$ & 8 & $>128$ & 8 & $>128$ & 2 & 32 & 2 & $>128$ & $<0.125$ & $<0.125$ & $<0.125$ \\
\hline J53-6 & NDM-1 & $>128$ & 8 & $>128$ & 8 & $>128$ & 2 & 32 & 4 & $>128$ & $<0.125$ & $<0.125$ & 0.25 \\
\hline
\end{tabular}

${ }^{a} S C F$, sulbactam/cefoperazone; $I P M$, imipenem; $C A Z$, ceftazidime; $M E M$, meropenem; $T Z P$, piperacillin /tazobactam; $P B$, polymixin $B ; F E P$, cefepime; $A M$, amikacin; $P I P$, piperacillin; $L E$, levofloxacin; $T G$, tigecycline; $A T M$, aztreonam 

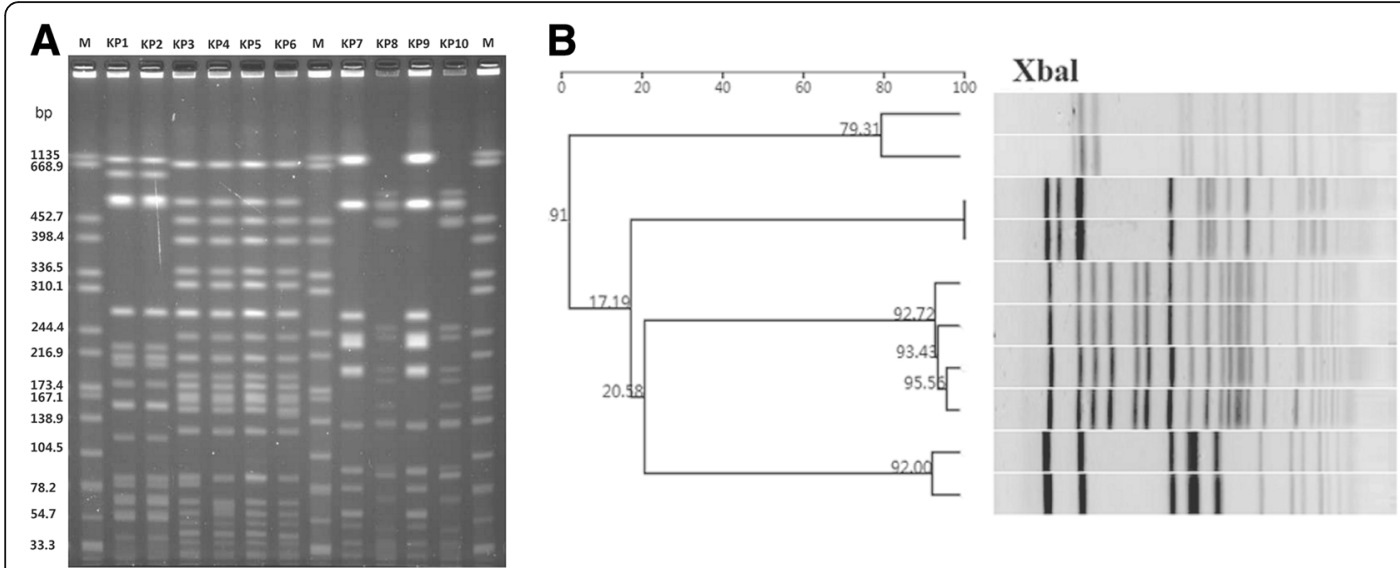

\begin{tabular}{|c|c|c|}
\hline \multicolumn{3}{|l|}{ KP8 } \\
\hline \multicolumn{3}{|l|}{ KP10 } \\
\hline KP1 & A & 234 \\
\hline KP2 & A & 234 \\
\hline KP6 & B & 1412 \\
\hline KP4 & B & 1412 \\
\hline KP3 & B & 1412 \\
\hline KP5 & B & 1412 \\
\hline \multicolumn{3}{|l|}{ KP7 } \\
\hline KP9 & & \\
\hline
\end{tabular}

Fig. 1 Pulsed-field gel electrophoresis analysis of isolated NDM-1- producing K. pneumoniae strains. A, Pulsed-field gel electrophoresis fingerprint of the isolates; B, Molecular epidemiology investigation of the isolates; KP1 to KP6: patients; KP7: incubator surface; KP 8: head circumference tape; KP 9: blood machine surface; KP 10: healthcare worker; lane M: marker (Salmonella H9812)

that all transconjugants displayed carbapenem resistance (Table 2). No other resistance genes were detected. The conjugation experiments were conducted multiple times with KP1 and KP2, but the results were always negative. All transconjugants revealed similar antimicrobial susceptibility as that of the donor strains.

\section{Plasmid analysis}

S1-PFGE and Southern blot hybridization analysis indicated that the $b l a_{\mathrm{NDM}-1}$ genes were transferred via the same plasmids (with approximate size $50 \mathrm{~kb}$ ), except for in the KP1 and KP2 strains (Fig. 2b). Although the KP1 and KP 2 strains carried plasmid DNA (with approximate size $110 \mathrm{~kb}$ ) (Fig. 2a), they did not seem to transmit the $b l a_{\mathrm{NDM}-1}$ gene via these plasmids (Fig. 2b). Additionally, conjugation experiments and Southern blotting hybridization showed that the $b l a_{\mathrm{NDM}-1}$ gene probably falls within the chromosome of KP1 and KP2 isolates.

\section{Discussion}

Infectious diseases caused by NDM-1-producing Enterobacteriaceae are known to be associated with high morbidity and mortality worldwide. This is a great challenge

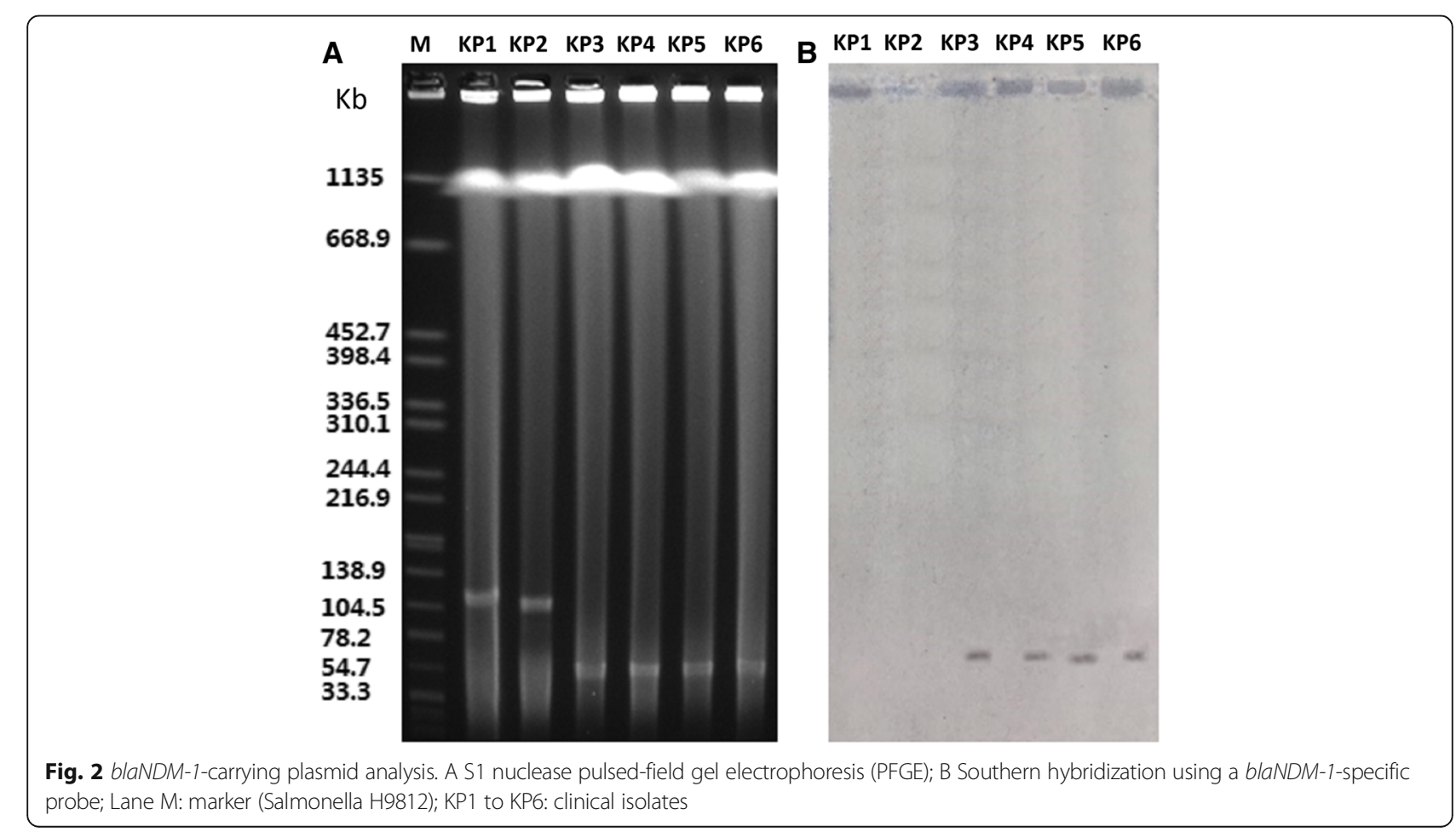


for paediatricians due to the transferability of the gene and the poor prognosis in children. Of the carbapenem resistance genes found in carbapenem-resistant Enterobacteriaceae in children, NDM genes (including coding genes of NDM-1 and NDM-6) are more common than KPC and VIM [10]. Compared to adults, children are more limited in antibiotic use. Although these isolates were all susceptible to tigecycline and colistin in vitro, the paediatrician had not used either of these treatments because of their potential side effects in children. Aminoglycosides and fluoroquinolones are also not used for children due to their nephrotoxicity and ototoxicity [6]. After careful consideration, the paediatrician in this report chose to use an increased dose of carbapenems (meropenem $0.5 \mathrm{~g}$ IVD qd; imipenem $1.0 \mathrm{~g}$ IVD qd), and no side effects were reported during the antimicrobial therapy. Fortunately, all of the neonates in this report had positive prognoses. However, the proposed doses of meropenem and imipenem for neonates who are greater than 28-days-old are $120 \mathrm{mg} / \mathrm{kg} /$ day and $60-100 \mathrm{mg} / \mathrm{kg} /$ day, respectively, according to the Sanford Guide to Antimicrobial Therapy. In the present study, the doses used are more than 4 times the amount suggested by the guide. Thus, a recent study showed that monotherapy for treatment of CRE infection in adults was associated with higher mortality than that of combination therapy, and limited data were available for children [10, 20, 21]. Although we had a good prognosis this time, further dosage optimization of carbapenem and medication for antimicrobial therapy for NDM-1-producing Enterobacteriaceae should be investigated.

In our study, PFGE analysis showed two clusters of the NDM-1-producing strains that corresponded to ST234 and ST1412. Among these, none were found to be clonally related to the carbapenem-susceptible $K$. pneumoniae isolated from the surface of incubators, blood machines, neonatal head circumference tapes and medical workers. Regarding the epidemiology of this spread, 2 isolates $(5,6)$ were identified in June, and 4 isolates $(1,2,3$, and 4$)$ were identified in August. This means that the ST1412 isolates remained in the neonatal ward for 2 months and reappeared in August, along with a new strain. It is unfortunate that although we have not found the source of this outbreak, we cannot deny that the hospital environment was likely the source of these infections. After all, the incubator water and the sharing of breast milk has been reported to be 'hotspots' for bacterial transmission in neonatal wards $[7,22]$. When we first confirmed the outbreak, our hospital control team took active measures to sterilize the source of infection in the environment and restrict the admission of newborns to the unit. Finally, the outbreak event was successfully controlled and all infected patients recovered and were discharged. NDM-1-producing $K$. pneumonia have been reported as belonging to various types of MLST:
ST11, ST14, ST17, ST25, ST37, ST76, ST105, ST147, ST149, ST231, ST340, and ST1043 [6, 7, 23]. Our data indicate that NDM-1-producing $K$. pneumoniae strains belong to two independent types, ST234 and ST1412, which differ from most types reported previously, particularly for the never reported NDM-1 producing ST234 strains, which have only been reported as KPC-producing $K$. pneumonia [24].

The plasmid analysis in this study showed that the $b l a_{\mathrm{NDM}-1}$ genes transferred using the same plasmids (with approximate size $50 \mathrm{~kb}$ ), except for the KP1 and KP2 strains. We analysed the genomic DNA of four transconjugants using PCR, and the results showed that only $b l a_{\mathrm{NDM}-1}$ was present. This result indicates that $b l a_{\mathrm{SHV}-148}$ was not on the same plasmid as $b l a_{\mathrm{NDM}-1}$. For the KP1 and KP2 strains, we speculated that the $b l a_{\mathrm{NDM}-1}$ gene may be in the chromosome or may be a high-molecular-weight non-conjugative plasmid that was not detected by the methodology used here. Further studies should be investigate this gene. The source of the NDM-1 has not been determined. It has been studied in previous $b l a_{\mathrm{NDM}}$ variants from sewage water in hospitals, which serves as an important source of the spread of antibiotic resistance genes [25]. Guidance from the European Centre for Disease Prevention and Control suggested that intervention measures should be taken on antimicrobial stewardship, clean hospital settings, equipment reprocessing, staff education and microbiological capacity to minimize risks of spread of CRE [26]. All we can do is strengthen the unity of the clinical departments to prevent further infection.

\section{Conclusions}

ST234 and ST1412 of NDM-1-producing Klebsiella pneumoniae are the resistant clone spread in the neonatal unit, comprehensive infection control measures and optimized carbapenem therapy played an important role in controlling this NDM-1-producing $K$. pneumoniae outbreak.

\section{Abbreviations \\ CLSI: The Clinical and Laboratory Standards Institute; CRE: Carbapenem resistant Enterobacteriaceae; CRKP: Carbapenem-resistant Klebsiella pneumoniae; ESBL: Extended-spectrum $\beta$-lactamase; MIC: Minimum inhibitory concentration; MLST: Multilocus sequence typing; PCR: Polymerase chain reaction; PFGE: Pulsed-field gel electrophoresis}

\section{Acknowledgements}

We would like to thank professor Wenen Liu (Zhongnan University) and Huimin Qian (Jiangsu Provincial Center for Disease Control and Prevention) for kindly provide the reference strains.

\section{Funding}

This study was supported by the Project of the Key Laboratory for Laboratory Medicine of Jiangsu Province (ZDXKB2016005), the innovation project for postgraduate education of Jiangsu Province(No. SJLX15_0436). None of these funding bodies had any in role in the design of the study and collection, analysis, and interpretation of data and in writing this manuscript. 


\section{Availability of data and materials}

All the data supporting our findings is contained within the manuscript. The Raw data and scientific records are saved in our department and can get from the corresponding author on reasonable request.

\section{Authors' contributions}

GYL, XH and XJC made substantial contributions to conception and acquisition of data, or analysis and interpretation of data; XH, XJC, PFS and CJT performed most of the research experiments; $\mathrm{XH}$ and CJT are involved in drafting the manuscript, GYL and FN revised it critically for important intellectual content; All the authors given final approval of the version to be published and agreed to be accountable for all aspects of the work.

\section{Ethics approval and consent to participate}

The study was approved by the Ethical Committee of the First Affiliated Hospital with Nanjing Medical University (2016-SRFA-162), The need for consent was waived in this vote.

\section{Consent for publication}

Not applicable.

\section{Competing interests}

The authors declare that they have no competing interests.

\section{Publisher's Note}

Springer Nature remains neutral with regard to jurisdictional claims in published maps and institutional affiliations.

Received: 27 April 2018 Accepted: 1 November 2018

\section{Published online: 14 November 2018}

\section{References}

1. Friedman ND, Carmeli Y, Walton AL, Schwaber MJ. Carbapenem-resistant Enterobacteriaceae: a strategic roadmap for infection control. Infect Control Hosp Epidemiol. 2017:38(5):580-94.

2. $\mathrm{Xu} \mathrm{L}$, Sun $X, M a X$. Systematic review and meta-analysis of mortality of patients infected with carbapenem-resistant Klebsiella pneumoniae. Ann Clin Microbiol Antimicrob. 2017;16(1):18

3. Ye Y, Xu L, Han Y, Chen Z, Liu C, Ming L. Mechanism for carbapenem resistance of clinical Enterobacteriaceae isolates. Exp Ther Med. 2018;15(1):1143-9.

4. Yong D, Toleman MA, Giske CG, Cho HS, Sundman K, Lee K, Walsh TR. Characterization of a new metallo-beta-lactamase gene, Bla (NDM-1), and a novel erythromycin esterase gene carried on a unique genetic structure in Klebsiella pneumoniae sequence type 14 from India. Antimicrob Agents Chemother. 2009:53(12):5046-54.

5. Khan AU, Maryam L, Zarrilli R. Structure, Genetics and Worldwide spread of New Delhi Metallo-beta-lactamase (NDM): a threat to public health. BMC Microbiol. 2017;17(1):101.

6. Zhu J, Sun L, Ding B, Yang Y, Xu X, Liu W, Zhu D, Yang F, Zhang H, Hu F. Outbreak of NDM-1-producing Klebsiella pneumoniae ST76 and ST37 isolates in neonates. Eur J Clin Microbiol Infect Dis. 2016;35(4):611-8.

7. Zheng R, Zhang Q, Guo Y, Feng Y, Liu L, Zhang A, Zhao Y, Yang X, Xia X. Outbreak of plasmid-mediated NDM-1-producing Klebsiella pneumoniae ST105 among neonatal patients in Yunnan, China. Ann Clin Microbiol Antimicrob. 2016:15:10.

8. Zhang X, Li X, Wang M, Yue H, Li P, Liu Y, Cao W, Yao D, Liu L, Zhou X, et al. Outbreak of NDM-1-producing Klebsiella pneumoniae causing neonatal infection in a teaching Hospital in Mainland China. Antimicrob Agents Chemother. 2015;59(7):4349-51.

9. Datta S, Mitra S, Chattopadhyay P, Som T, Mukherjee S, Basu S. Spread and exchange of Bla NDM-1 in hospitalized neonates: role of mobilizable genetic elements. Eur J Clin Microbiol Infect Dis. 2017;36(2):255-65.

10. Logan LK. Carbapenem-resistant enterobacteriaceae: an emerging problem in children. Clin Infect Dis. 2012:55(6):852-9.

11. CLSI, editor. Performance Standards for Antimicrobial Susceptibility Testing, vol. CLSI supplement M100S. 26th ed. Wayne: Clinical and Laboratory Standards Institute; 2016

12. European Committee on Antimicrobial Susceptibility Testing Available at: http://www.eucast.org
13. Liao XP, Xia J, Yang L, Li L, Sun J, Liu YH, Jiang HX. Characterization of CTXM-14-producing Escherichia coli from food-producing animals. Front Microbiol. 2015;6:1136

14. Chen $L$, Chen ZL, Liu JH, Zeng ZL, Ma JY, Jiang HX. Emergence of RmtB methylase-producing Escherichia coli and Enterobacter cloacae isolates from pigs in China. J Antimicrob Chemother. 2007:59(5):880-5.

15. Cattoir V, Poirel L, Rotimi V, Soussy CJ, Nordmann P. Multiplex PCR for detection of plasmid-mediated quinolone resistance qnr genes in ESBLproducing enterobacterial isolates. J Antimicrob Chemother. 2007;60(2):394-7.

16. Diancourt L, Passet V, Verhoef J, Grimont PA, Brisse S. Multilocus sequence typing of Klebsiella pneumoniae nosocomial isolates. J Clin Microbiol. 2005; 43(8):4178-82.

17. Huang $Y M$, Zhong $L L$, Zhang $X F$, Hu HT, Li YQ, Yang XR, Feng $L Q$, Huang $X$ Tian GB. NDM-1-producing Citrobacter freundii, Escherichia coli, and Acinetobacter baumannii identified from a single patient in China. Antimicrob Agents Chemother. 2015;59(8):5073-7.

18. Tenover FC, Arbeit RD, Goering RV, Mickelsen PA, Murray BE, Persing DH, Swaminathan B. Interpreting chromosomal DNA restriction patterns produced by pulsed-field gel electrophoresis: criteria for bacterial strain typing. J Clin Microbiol. 1995;33(9):2233-9.

19. Carattoli A, Bertini A, Villa L, Falbo V, Hopkins KL, Threlfall EJ. Identification of plasmids by PCR-based replicon typing. J Microbiol Methods. 2005:63(3):219-28.

20. Morrill HJ, Pogue JM, Kaye KS, LaPlante KL. Treatment options for Carbapenem-resistant Enterobacteriaceae infections. Open forum infectious diseases. 2015;2(2):ofv050.

21. Tzouvelekis LS, Markogiannakis A, Piperaki E, Souli M, Daikos GL. Treating infections caused by carbapenemase-producing Enterobacteriaceae. Clin Microbiol Infect. 2014;20(9):862-72.

22. Nakamura K, Kaneko M, Abe Y, Yamamoto N, Mori H, Yoshida A, Ohashi K, Miura S, Yang TT, Momoi N, et al. Outbreak of extended-spectrum betalactamase-producing Escherichia coli transmitted through breast milk sharing in a neonatal intensive care unit. J Hosp Infect. 2016;92(1):42-6.

23. Giske CG, Froding I, Hasan CM, Turlej-Rogacka A, Toleman M, Livermore D, Woodford N, Walsh TR. Diverse sequence types of Klebsiella pneumoniae contribute to the dissemination of blaNDM-1 in India, Sweden, and the United Kingdom. Antimicrob Agents Chemother. 2012;56(5):2735-8.

24. Chen L, Chavda KD, Melano RG, Jacobs MR, Koll B, Hong T, Rojtman AD, Levi MH, Bonomo RA, Kreiswirth BN. Comparative genomic analysis of KPCencoding pKpQIL-like plasmids and their distribution in New Jersey and New York hospitals. Antimicrob Agents Chemother. 2014;58(5):2871-7.

25. Parvez S, Khan AU. Hospital sewage water - a reservoir for variants of New Delhi metallo-beta-lactamase (blaNDM) and ESBL-producing enterobacteriaceae. Int J Antimicrob Agents. 2018;51(1):82-8.

26. Magiorakos AP, Burns K, Rodriguez Bano J, Borg M, Daikos G, Dumpis U, Lucet JC, Moro ML, Tacconelli E, Simonsen GS, et al. Infection prevention and control measures and tools for the prevention of entry of carbapenemresistant Enterobacteriaceae into healthcare settings: guidance from the European Centre for Disease Prevention and Control. Antimicrob Resist Infect Control. 2017:6:113.
Ready to submit your research? Choose BMC and benefit from:

- fast, convenient online submission

- thorough peer review by experienced researchers in your field

- rapid publication on acceptance

- support for research data, including large and complex data types

- gold Open Access which fosters wider collaboration and increased citations

- maximum visibility for your research: over $100 \mathrm{M}$ website views per year

At BMC, research is always in progress.

Learn more biomedcentral.com/submission 